\title{
La traducción alemán-español de textos médico-jurídicos y su utilidad didáctica: el consentimiento informado
}

\author{
German to Spanish translation of medico-legal documents \\ and its teaching utility: Informed consent form
}

\author{
Isidoro Ramírez Almansa
}

Universidad de Córdoba.112raali@uco.es

Recibido: 01.05.2019. Aceptado: 08.09.2019

Resumen: El presente trabajo aborda el estudio del consentimiento informado (CI) y su traducción en la combinación de lenguas alemán-español. Para ello, analizaremos el género textual médico-jurídico y qué tipologías textuales lo conforman. Una vez definido el CI, nos centraremos en su estudio en investigación clínica, y mostraremos su utilidad para la enseñanza de la traducción especializada. Para ello, realizaremos un análisis desde un punto de vista contrastivo de la estructura y la terminología presente en un CI original alemán. Esto nos permitirá determinar, si las hubiere, las diferencias con el CI español, así como aportar soluciones a los problemas de traducción que pudiesen surgir.

Palabras clave: documento de consentimiento informado; didáctica de la traducción; terminología medico-jurídica; investigación clínica; texto híbrido.

\begin{abstract}
This study focuses on the informed consent form (ICF) and its German-Spanish translation. To this end, the medico-legal text genre and the different texts that belong to it are analysed. Subsequently, we aim to analyse the informed consent form in clinical research, as well as we prove its educational utility applied to specialized translation. Thus, we carry out a contrastive analysis of the structure and terminology from an original informed consent form originally written in German. This helps us establish the differences between an informed consent form in a German-Spanish version, in case, and also find a solution to the translation problems which may arise.
\end{abstract}

Keywords: informed consent form; translation teaching; medico-legal documents; clinical research; hybrid text.

》) Ramírez Almansa, Isidoro. 2019. "La traducción alemán-español de textos médicojurídicos y su utilidad didáctica: el consentimiento informado". Quaderns de Filologia: Estudis Lingüistics XXIV: 229-245. doi: 10.7203/QF.24.16306 



\section{Introducción}

La traducción del CI no ha suscitado demasiado interés entre los investigadores, siendo destacable en nuestro país los trabajos de Martínez (2009a), Borja (2012), Gallardo (2012), Pajares (2015) o GallegoBorghini (2015), entre otros. Todos ellos, no obstante, se ocupan de la traducción en el par de lenguas inglés-español ${ }^{1}$.

En lo relativo a la traducción biosanitaria alemán-español, en los últimos años sí se ha producido un avance en la creación de recursos para la traducción biosanitaria, entre los que cabe señalar el Gran diccionario médico alemán-español Medizin (2017) que ya va por su segunda versión 1.04 actualizada en noviembre de 2018, o el Glosario (alemánespañol) de términos relacionados con el sistema sanitario alemán de Parcet (2018).

Partiendo de esta situación inicial, pretendemos en este trabajo contribuir a paliar la escasez de estudios en torno a la traducción del CI en la combinación lingüística alemán-español, y a ello dedicaremos las páginas siguientes.

\section{2. ¿Textos médico-legales o médico-jurídicos?}

Podemos encontrar distintas denominaciones para referirse al grupo de textos del que forma parte el CI; hay autores como Martínez (2009a, 2009 b y 2009c), Casado (2009) o Gallardo (2012) que los denominan textos médico-legales; otros como Borja (2012), Gallego-Borghini (2015) o Pajares (2015) hablan de textos médico-jurídicos. Para establecer una diferencia entre ambas denominaciones recurrimos a Rodes (2014: 2), que define la medicina legal como:

Ciencia social que aplica conocimientos médicos a la resolución de problemas jurídicos. Dicho de otra forma: es la ciencia que tiene por objeto el estudio de las cuestiones que se presentan en el ejercicio profesional

\footnotetext{
${ }^{1}$ Coincidimos con Martínez (2009a: 37) en que los estudios centrados en estas combinaciones de lengua se deben, precisamente, a la condición de lingua franca del inglés en el ámbito biosanitario. En este sentido, destacamos igualmente que otros autores han desarrollado recursos para el traductor de CI en inglés y español (Mugüerza et alii, 2010 y Saladrigas, 2019).
} 
del jurista y cuya resolución se funda total o parcialmente en ciertos conocimientos médicos o biológicos previo.

En nuestro estudio adoptaremos la denominación de textos médico-jurídicos, pues deseamos primar ante todo el valor jurídico de los textos que engloban la categoría de los textos médico-jurídicos, y entendemos que estos pueden ser legales o no dependiendo de si cumplen o incumplen la ley. Para esta distinción hemos tomado como referencia el Diccionario del español jurídico (DEJ) y su definición de legal: Adecuado o conforme a la ley. Por lo que consideramos que la medicina legal tan solo abarcaría parte y no la totalidad de los textos médico-jurídicos. Por ello, nos decantamos por jurídico frente a legal, lo que no significa que no contemplemos aquellos trabajos que sí han optado por la opción contraria, dado que sí han favorecido el avance del campo que nos ocupa.

\subsection{Los textos médico-jurídicos}

Podemos encontrar varias definiciones de textos médico-jurídicos, Casado (2008: 19) los define como:

Todas aquellas actuaciones escritas que utiliza el médico en sus relaciones profesionales con las autoridades, los organismos y las instituciones o con cualquier persona.

Con ello, concibe al médico como la única fuente de la que emanan este tipo de textos, aspecto este quizá debido a que el autor es médico y, por tanto, ha elaborado la definición desde el punto de vista del facultativo. Consideramos que la definición propuesta por Borja (2012: 167) es más adecuada para los objetivos de nuestro estudio:

Aquellos [textos] en los que aparecen combinados conceptos propios de la medicina y conceptos legales [...], o bien textos médicos que pueden surtir efectos legales.

Ya en la definición aparece, de manera indirecta, el concepto de hibridez que caracteriza a este tipo de textos, dado que en ellos conviven dos disciplinas, la primera de naturaleza jurídica y la segunda de naturaleza médica. La definición mencionada forma parte de aquellos estudios en 
los que se emplea el adjetivo legal, contradiciendo nuestra propuesta. No obstante, observamos que dicha definición se elaboró concibiendo legal en su segunda acepción (Perteneciente o relativo a la ley o al derecho) recogida en la versión electrónica 23.2 del Diccionario de la lengua española ( $D L E)$ cuyo uso no hemos contemplado, debido a que el $D L E$ no es un diccionario especializado en derecho, aunque sospechamos que sí fue el uso empleado por la autora en su momento al contemplar jurídico y legal como sinónimos.

Martínez (2009a: 45) pone también de manifiesto el carácter híbrido de este tipo de textos, y considera que no es posible "hacer una caracterización única" debido a que conviven diferentes "tipos de textos y/o prototipos textuales" que en un mayor o menor porcentaje presentan rasgos propios del texto jurídico y del texto biosanitario. Basándonos en su clasificación podemos distinguir cinco grandes grupos de textos:

1. El documento médico-legal emanado de la actuación de un médico (generalista, por lo general) o de un médico forense: certificado médico oficial, certificado de nacimiento o defunción, etc. En estos textos están presentes más características de los textos jurídicos que científicos.

2. El documento médico-legal para fines judiciales: el especialista manifiesta su opinión documentada acerca del estado de salud de un paciente. Este grupo de textos son cercanos al informe técnico o científico, pese a que forman parte de un proceso administrativo o judicial.

3. El consentimiento informado: se define como el tipo de documento que presenta una mayor hibridez. Guarda un alto grado de verosimilitud con un contrato entre dos partes contratantes: la parte médica y el paciente. Su estructura recuerda a la de un texto jurídico-administrativo.

4. El informe divulgativo sobre prácticas o tratamientos sanitarios específicos: estos textos son los menos híbridos y en ellos se explica la puesta en práctica de una actividad sanitaria específica. No obstante, la legislación está presente con el fin de informar al paciente.

5. El informe técnico de naturaleza farmacológico-legal: este tipo de documento guarda cierta similitud con el informe pericial en cuanto al contenido, aunque no en la estructura. Respecto a esta, 
siempre presenta una serie de secciones fijadas por las autoridades sanitarias de cada país. Su contenido es altamente científico.

Por último, Borja (2012: 165-175) sigue en la línea de Martínez y divide los textos médico-jurídicos en cinco grandes grupos según la situación comunicativa: textos normativos de temática médica, textos judiciales de temática médica, acuerdos de voluntades o contratos, declaraciones unilaterales de voluntad y textos administrativos que requieren la intervención de un médico para tener eficacia jurídica o resoluciones administrativas sobre aspectos de salud.

Tomamos estas dos últimas clasificaciones como las más adecuadas para nuestro estudio, en tanto que se basan en la situación comunicativa.

\subsection{El consentimiento informado como género textual}

El CI es un documento en el que podemos diferenciar dos partes: la hoja de información para el paciente (HIP) y la declaración de consentimiento informado. Esta segunda parte no debe confundirse con el CI propiamente dicho que se refiere a las dos partes, pese a su parecido en la denominación. Gallego-Borghini (2015a: 11) determina que el CI suele tener una longitud de 10-12 hojas y afirma que consta de alrededor de unas 4000-5000 palabras. En febrero de 2018, la Agencia Española de Medicamentos y Productos Sanitarios (en adelante, AEMPS) publicó la Guía para la correcta elaboración de un modelo de hoja de información al paciente y consentimiento informado (HIP/CI), en la que determina que el CI no debe sobrepasar las 15 páginas, por lo que recomienda una extensión menor de la que suelen tener los CI en la realidad de acuerdo a Gallego-Borghini. Suponemos que dicha recomendación de la AEMPS viene motivada con el fin de hacer los CI más breves, claros y concisos para aumentar su nivel de legibilidad de cara a su lectura por el paciente.

En lo referente a la HIP, esta debe contener los siguientes apartados: a) introducción, en la que se presente el estudio y se declare que cumple con la ley vigente habiendo sido revisado por la AEMPS; b) participación voluntaria; c) objetivo del estudio; d) descripción del estudio; e) actividades del estudio; f) riesgos y molestias derivados de su participación en el estudio; g) posibles beneficios; h) advertencia relativa al embarazo; i) tratamientos alternativos; j) seguro; k) protección de datos 
personales; 1) gastos y compensación económica; m) otra información relevante; n) ¿qué tratamiento recibiré cuando finalice el ensayo clínico?; o) contacto en caso de duda; p) estudios clínicos en menores de edad; q) obtención y utilización de muestras biológicas; r) cuando en el estudio principal se realice algún subestudio.

A continuación de la HIP encontraremos la hoja de consentimiento informado, a la que también podemos denominar formulario o documento de firma del consentimiento. Su longitud es más breve (desde una hasta 2-3 hojas) y tiene un carácter más legal que médico. Esta parte del CI tiene como fin dar fe legal del acuerdo entre las dos partes que participan en la investigación clínica: el médico y el paciente. En esta segunda parte, médico y paciente participan a partes iguales con su firma dando su conformidad legal. Al firmar este documento el paciente presta su conformidad al confirmar que: a) ha leído la hoja de información que se le ha entregado sobre el estudio; b) ha podido hacer preguntas sobre el estudio; c) ha recibido información suficiente sobre el estudio; d) ha hablado con el investigador principal; e) comprende que su participación es voluntaria; f) comprende que puede retirarse del estudio cuando quiera, sin tener que dar explicaciones y sin que su retirada repercuta en sus cuidados médicos. Cuando el paciente firma esta segunda parte del CI, recibe una copia firmada y sellada de todo el documento.

La macroestructura que acabamos de describir es el prototipo que pueden presentar las dos partes del CI. Esta estructura puede verse alterada en función del estudio que se vaya a realizar. Por ejemplo, si es necesario hacer análisis genéticos, si participan menores de 12-17 años, o si el estudio contempla la recogida de pruebas biológicas, tendrá que contener apartados relativos a estos aspectos.

\section{La traducción del consentimiento informado y su utilidad didáctica}

En este apartado de nuestro estudio analizaremos, en primer lugar, la importancia y funciones que desempeña el CI enmarcado en la legislación española y la investigación clínica. El CI ha sido estudiado desde diferentes puntos de vista por diversos perfiles profesionales como médicos o traductores. Asimismo, ofrecemos un análisis contrastivo DE-ES de su macroestructura y terminología en el que destacamos los 
problemas que pueden surgir al abordar su traducción y cómo podrían solucionarse.

\subsection{Implicaciones para su traducción y utilidad didáctica}

El CI es un instrumento para la comunicación entre médico y paciente, lo que hace a estas dos figuras las destinatarias únicas del texto, y su creación está motivada por motivos ético-jurídicos con el fin de asegurar la autonomía del paciente. El CI se ha convertido en un elemento indispensable dentro de la investigación clínica, "especialmente los ensayos clínicos con medicamentos" (Gallego-Borghini, 2015a: 1).

Uno de los aspectos que hemos de tener en cuenta a la hora de traducir el CI es que sus destinatarios son el médico y el paciente, siendo este último un usuario no especialista al que no debemos atribuir conocimientos técnicos como al médico. Esto hará que, a pesar de su carácter especializado y la presencia de terminología médica y legal, su lectura deba ser clara y concisa para que el paciente lo entienda. Si no, se estaría incumpliendo con uno de sus objetivos principales: informar al paciente de las pruebas a las que va a someterse y hacerle conocedor de sus derechos. Además, el CI, según Gallego-Borghini (2015: 2), también se concibe en cierta manera como una "guía o referencia" para que de esta forma el paciente o quien él decida consulte toda la información referente al estudio o a su participación en el mismo. De hecho, la AEMPS (2002: 20) apostilla al hablar sobre el CI:

El lenguaje utilizado en la información oral y escrita sobre el ensayo, incluyendo la hoja de información al sujeto, no deberá ser técnico sino práctico y deberá poder ser entendido por el sujeto o su representante legal y el testigo imparcial, cuando proceda.

Igualmente, si analizamos la presencia del CI en textos jurídicos observamos que el Real Decreto 223/2004 en su epígrafe 2 del artículo 7 versa:

El consentimiento informado [...] La hoja de información contendrá únicamente información relevante, expresada en términos claros y comprensibles para los sujetos [...]. 
El Real Decreto vuelve a hacer hincapié en la claridad y legibilidad del documento. Asimismo, el CI debe cumplir la ley vigente, las normas de Buena Práctica Clínica (BPC) y los principios éticos presentes en la Declaración de Helsinki promulgada por la Asociación Médica Mundial (AMM). Jurídicamente, el CI en España aparece recogido en el ordenamiento jurídico mediante la Orden de 3 de agosto de 1982 por la que se desarrolla el Real Decreto 944/1978 sobre ensayos clínicos en humanos. La denominación consentimiento informado no aparece como tal hasta el año 1993 con el Real Decreto 561/1993. Asimismo, el CI también aparece en el Real Decreto 223/2004 de 6 de febrero por el que se regulan los ensayos clínicos con medicamentos, así como las diferentes circulares y sus correcciones de la AEMPS, como la ya mencionada del año 2002. Este marco jurídico y administrativo en el que se enmarca el CI provoca que, ante una mala redacción o traducción del mismo, no solo tenga consecuencias directas para el paciente y el equipo que dirige la investigación, sino que además es un problema ético y legal. Hasta tal punto el valor ético del CI es importante que Galende (2007) manifiesta en su estudio que son los CEIC (Comité Ético de Investigación Clínica) los que en muchas ocasiones velan por corregir y volver a redactar las hojas de información de los CI para asegurar que se están cumpliendo los principios de lenguaje legible y no excesivamente técnico. Dal-Ré et alii (2004), en su trabajo, manifiestan que los CEIC, en más del $60 \%$ de las ocasiones, demandan que se haga alguna aclaración al CI. La función que desempeñan los CEIC en el CI y su redacción es mayor de la que podemos pensar a priori. Gallego Borghini (2015b), en su estudio, analizó 100 peticiones de los CEIC y determina que el $68 \%$ está relacionada con el CI y casi el $32 \%$ está relacionada con la traducción en términos de estilo o redacción del documento, o solicitando realizar algunas modificaciones en frases o apartados concretos.

En cuanto al potencial didáctico de la inclusión del CI en el aula de traducción, consideramos que es más que manifiesto. Martínez (2009a: 47) ya destacó este aspecto en su estudio, en el que defiende que este documento es susceptible de integrarse tanto en la asignatura de traducción jurídica como en la de traducción científica, a lo que nosotros añadimos también la asignatura de traducción biosanitaria que con la instauración del Plan Bolonia ya existe como tal a nivel de grado (Universidad de Córdoba, Rey Juan Carlos o Universidad Alfonso X 
El Sabio). Asimismo, consideramos que el carácter híbrido del CI lo convierte en uno de los mejores ejemplos para ilustrar un caso de traducción de textos híbridos, por lo que hacemos extensible también su utilidad en asignaturas de traducción general. En los últimos años, observamos cómo cada vez más la hibridez de los textos, en lo referente a su terminología o características de género textual, es motivo de estudio y discusión en los foros académicos y profesionales de la traducción. La traducción de textos médico-jurídicos, periodísticos, turísticos, agroalimentarios o la traducción audiovisual son ejemplos en los que la hibridez está presente; por ello, entendemos que es un elemento que debe recogerse en los planes de estudio de los grados en TeI.

Por su parte, Pajares (2015: 190) llevó a cabo un estudio sobre si la categoría de textos médico-jurídicos estaban presentes en la práctica profesional de la traducción. El investigador realizó una clasificación de 32 textos de esta categoría e hizo una encuesta en la que el $100 \%$ de los traductores profesionales participantes contestaron que sí se habían enfrentado a lo largo de su carrera profesional a la traducción de un texto de este tipo. Este dato apoya nuestra afirmación del valor didáctico del $\mathrm{CI}$ en el aula de traducción, ya que como docentes e investigadores de la traducción, uno de nuestros principales objetivos es ofrecer al alumnado una formación que lo convierta, al finalizar sus estudios, en un profesional competente y adaptado a las necesidades del mundo laboral.

\subsection{Análisis contrastivo DE-ES de la macroestructura y terminología del CI}

En esta parte de nuestro estudio llevaremos a cabo un análisis contrastivo alemán-español a partir de un documento de CI redactado originalmente en alemán. Para ello, hemos seleccionado un CI de un ensayo clínico llevado a cabo por la ESA (European Society of Anaesthiology) bajo el título Reevaluation von MET zur Abschätzung des perioperativen Herzrisiko (METREPAIR): eine prospektive, multizentrische Kohortenstudie.

Nuestro análisis contempla aspectos como las diferencias en la macroestructura del CI en alemán y español y su terminología. Con este fin nos hemos servido del estudio de Martínez (2009a: 47-49) en el que elabora una lista donde recoge las dificultades que deben tratarse en la clase de traducción al abordar la traducción del CI. Para ello se ha basa- 
do en un ejemplo real de CI sobre la donación de semen. Dicho estudio establece una serie de dificultades de las que destacamos las siguientes:

1. Localización y dificultades terminológicas del TO en lo que respecta a la identificación de las partes del CI y búsqueda de equivalencias en la LM, es decir, la traducción de unidades fraseológicas tales como firma del doctor, nombre y apellidos del paciente, nombre y apellidos del representante legal, etc.

2. Localización y dificultades terminológicas del TO (frases y expresiones hechas) y búsqueda de equivalencias en la LM: a los fines anteriormente citados, a rellenar por el donante, carácter anónimo y secreto de la donación, etc.

3. Localización de los referentes culturales y propuesta de traducción en la LM: Instituto Nacional de Salud o Ley 14/2006 sobre técnicas de reproducción asistida.

Hemos seleccionado estas dificultades, ya que las otras destacadas dependen de las características propias de cada CI.

Esta primera parte del análisis abarca las dificultades presentes en los puntos 2, 3 y 5 del estudio de Martínez (2009a: 47-49). En dicho análisis encontramos culturemas, como la Osterreichischen Datenschutzbehörde para la que proponemos la traducción Agencia austríaca de Protección de Datos (Österreichischen DSB), manteniendo el nombre de la institución en alemán en el TM. En lo referente a la protección de datos, también se hace referencia al Datenschutz-Grundverordnung (DSGVO), en español Reglamento General de Protección de Datos (RGPD) para referirse a la Ley Orgánica de Protección de Datos Personales y garantía de los derechos digitales. También encontramos numerosos ejemplos en los que el enunciado pasa a ser enunciativo en el TM: Was ist der Zweck der klinischen Studie? y Wie läuft die Klinische Studie? se traduce por Objetivo de la investigación/ estudio y Descripción del estudio / ensayo clínico. Asimismo, también encontramos el uso de Klinische Studie y Klinische Prüfung como sinónimos, también existente en español con ensayo clínico y estudio clínico, según Gallego-Borghini (2015a: 57) o Navarro (2010). En el texto en español observamos el empleo de las siglas HIP y CI, sin equivalencia en lengua alemana y cuyo uso es poco común enmarcado fuera del título de epígrafes y secciones. 
Esta primera parte de nuestro análisis ha puesto de relieve a su vez otro aspecto diferenciador del CI en ambas lenguas: su macroestructura. La organización del documento en español, tras ofrecer el nombre e información del estudio que se va a realizar, ofrece a continuación una serie de secciones que comienza por la introducción. Por el contrario, en alemán la información que en español se ofrece en la introducción se indica seguidamente tras ofrecer los datos del estudio. Es decir, en la macroestructura del texto en alemán no se contempla el apartado de introducción que sí se contempla en la convención española de este documento. Si seguimos analizando la macroestructura, es frecuente que, en español, entre el punto Objetivos del estudio y su descripción se incluya un apartado denominado Participación voluntaria o criterios de admisión en el que se le explica al paciente por qué se le invita a participar en el estudio y que lo hace de forma voluntaria. De hecho, este apartado tiene una estructura fija en español, aunque en los textos analizados en lengua alemana no es así, y se incluye en el primer apartado sin enumerar junto con la información que en el formato español se indica en la introducción. Estas diferencias demuestran que sin importar si el CI está escrito en lengua española o alemana siempre incluye la misma información, pero su organización para presentarla sí es diferente.

\section{Conclusiones}

Tras realizar un análisis contrastivo del CI alemán-español y estudiar los resultados obtenidos, llegamos a la conclusión de que el CI es un documento bastante normalizado en ambas lenguas, dado su valor jurídico. No obstante, existen ciertas diferencias en su contenido y en su forma. Es frecuente que en los títulos de los epígrafes en alemán el uso de oraciones interrogativas (Wie läuft die Klinische Studie?), lo que difiere de la convención en español que opta por enunciados afirmativos (Descripción del estudio). Además, no basta con modificar el tipo de enunciado para cumplir la convención, sino también la forma en la que cada idioma designa cada epígrafe puede ser totalmente diferente (Möglichkeit zur Diskussion weiterer Fragen en alemán y Contacto en caso de dudas en alemán).

Asimismo, llama también la atención como en alemán ni siquiera existen siglas para referirse a la hoja de información para el paciente (HIP) y consentimiento informado (CI). No obstante, en español el uso 
de estas siglas queda reservado para usos muy concretos y puntuales. Consideramos también importante pues destacar que como docentes de traducción debemos enseñar a reflejar en nuestro TM la convención del CI en español, lo cual no implica que el traductor pueda añadir en su traducción algo que no esté en el original, es decir, no puede existir algo en el TM si no está presente en el TO, sino que se debe expresar el contenido del TO en su forma convencionalizada. Como ejemplo para ilustrar esta tesitura a la que puede enfrentarse el traductor DE-ES nos remitimos a la macroestructura del texto, en el caso de CI en alemán no existe el apartado de Introducción, sino que esa información se ofrece justo debajo del nombre del estudio. Ante esta coyuntura debemos respetar siempre la macroestructura del TO, por lo que si traducimos el texto del alemán al español nuestro texto no contendría un apartado denominado Introducción, ya que no podemos crear en la versión del TM algo que no existe en el TO. Siguiendo esta metodología, el apartado en alemán Wann wird die klinische Studie vorzeitig beendet? se traduciría de acuerdo con la convención en español, pero se insertaría en nuestro TM siguiendo la macroestructura del TO.

Igualmente, defendemos la utilidad didáctica del CI en la clase de traducción, no solo por su importancia en el mundo laboral de los futuros egresados, sino también por las competencias que se trabajan. El CI podría insertarse en la programación de varias asignaturas (traducción jurídica, científica o biosanitaria) debido a su terminología y temática. Asimismo, el CI sirve como perfecto ejemplo de texto en las clases de traducción general o de fundamentos de la traducción, ya que con él se trabaja la metodología de trabajo de la combinación lingüística alemánespañol que se guía por las convenciones del género, al contrario del inglés que se guía en mayor medida por la tipología textual. De esta forma el alumnado aprenderá cómo resolver problemas relacionados con las diferencias entre alemán y español en la macroestructura y convenciones del texto.

También, manifestamos que la presencia de textos híbridos en la clase de traducción es necesaria dada la ingente cantidad de traducciones híbridas que existen en la práctica profesional más allá de la traducción de textos médico-jurídicos. Podemos destacar entre otras traducciones híbridas, la audiovisual, la turística o la periodística. Por último, tan solo expresar que con este estudio pretendemos que se avance en la traducción DE-ES del CI sin más pretensión que la de elaborar y ofrecer 
un estudio que debe ser ampliado, dado que no hemos podido abarcar el CI en su totalidad por razones obvias. Por ello, sería necesario seguir profundizando en su terminología y macroestructura con la elaboración de un mayor corpus textual que aporte un mayor grado de representatividad al estar conformado por ejemplos de CI en los que haya involucrados menores, obtención y manipulación de muestras biológicas o estudios en los que también se abarquen subestudios.

\section{Bibliografía}

AEMPS. 2008. Normas de buena práctica clínica. Madrid: AEMPS. https:// www.aemps.gob.es/industria/inspeccionBPC/docs/guia-BPC octubre-2008.pdf [Acceso 15/03/2019].

AEMPS. 2018. Guía para la elaboración de un modelo de hoja de información al paciente y consentimiento informado (HIP/IC). Madrid: AEMPS. https://www.aemps.gob.es/investigacionClinica/medicamentos/docs/ anexo8a-Ins-AEMPS-EC.pdf [Acceso 10/03/2019].

AEMPS. 2019. Documento de instrucciones de la Agencia Española de Medicamentos y Productos Sanitarios para la realización de ensayos clínicos en España. Madrid: AEMPS. https://www.aemps.gob.es/ investigacionClinica/medicamentos/docs/Instrucciones-realizacionensayos-clinicos.pdf [Acceso 10/03/2019].

Boletín Oficial del Estado. Número 166.13 de julio de 1993. Madrid: BOE. https://www.boe.es/eli/es/rd/2004/02/06/223/con [Acceso 12/03/2019].

Boletín Oficial del Estado. Número 294. 6 de diciembre de 2018. Madrid: BOE. https://www.boe.es/boe/dias/2018/12/06/pdfs/BOE-A-2018-16673.pdf [Acceso 18/09/2019].

Boletín Oficial del Estado. Número 33. 7 de febrero de 2004. Madrid: BOE. https://www.boe.es/eli/es/rd/2004/02/06/223/con [Acceso 12/03/2019].

Borja Albi, Anabel. 2012. Aproximación traductológicas a los textos médico-jurídicos.Panace@: Revista de Medicina,Lenguaje y Traducción 13(36): 167-175. http://www.medtrad.org/panacea/IndiceGeneral/n36tradyterm_ABorjaAlbi.pdf [Acceso 20/02/2019].

Borja Albi, Anabel \& Gallego-Borghini, Lorenzo. 2012. Los género médicojurídicos. Textos híbridos en la confluencia de dos grandes disciplinas. Panace@: Revista de Medicina, Lenguaje y Traducción 13(36): 163165. http://www.medtrad.org/panacea/IndiceGeneral/n36-editorial.pdf [Acceso 25/02/2019].

Casado Blanco, Mariano. 2008. Manual de documentos médico-legales. España: Consejería de Sanidad y Dependencia de la Junta de Extremadura. 
Cecilia, Ramos, Luciana. 2012. El consentimiento informado. Panace@: Revista de Medicina, Lenguaje y Traducción 13(36): 294-298. http:// www.medtrad.org/panacea/IndiceGeneral/n36-revistilo_LCRamos.pdf [Acceso 25/02/2019].

Dal-Ré, Rafael, Morejón, Elena \& Ortega Rafael. 2004. Nature and extent of changes in the patient's information sheets of international multicentre clinical trials as requested by Spanish Research Ethics Committees. Medicina Clínica 123: 770-774. doi: 10.1016/S0025-7753(04)74663-1

Dragan, Adriana. 2009. The importance of addressing linguistic ethno-cultural diversity in the delivery of public health services: a literature review. Canadá: Region of Peel Public Health.

Gallardo, San Salvador, Natividad. 2012. Hacia una "globalización " de la traducción: El caso de la traducción jurada de los documentos médicolegales (inglés-español). Sendebar 23: 227-250. http://revistaseug.ugr. es/index.php/sendebar/article/view/37/37 [Acceso 02/03/2019].

Galende Domínguez, Inés. 2007. Listas guía para la evaluación de protocolos de investigación clínica. En Galende Domínguez, Inéz (ed.) Guías operativas para los CEI-II: evaluación de protocolos de investigación biomédica. Madrid: Fundación AstraZeneca.

Gallego Borghini, Lorenzo. 2015a. La traducción inglés-español del consentimiento informado en investigación clínica. Barcelona: Fundación Dr. Antonio Esteve.

Gallego Borghini, Lorenzo. 2015b. Objeciones planteadas por los comités éticos a las traducciones de los documentos de consentimiento informado en la investigación clínica: análisis e implicaciones para el trabajo del traductor. Revista de Bioética y Derecho 33: 14-27. doi: 10.1344/ rbd2015.33.11561

Gálvez Múgica, Mari Ángeles \& De Pablo López de Abechuco, Itziar. 2007. El proceso de evaluación de un ensayo clínico desde la perspectiva de un comité ético de investigación clínica. Revista Clínica Española 207: 29-33. https://www.revclinesp.es/es-el-proceso-evaluacion-un-ensayoarticulo-13098498 [Acceso 20/03/2019].

López-Parra, María, Moreno-Quiroga, Coloma \& Lechuga Pérez, Javier. 2012. Revisión de las observaciones más frecuentes en la hoja de información al paciente para ensayos clínicos. Medicina Clínica 139: 176-179. doi: 10.1016/j.medcli.2012.02.011

Martínez-López, Ana Belén. 2009a. Sobre la traducción de textos médicolegales. redit 2: 33-52. doi: http://dx.doi.org/10.24310/REDIT.2009. v0i2.1906

Martínez-López, Ana Belén. 2009b. Terminología y fraseología en los documentos médico-legales (1): extracción, clasificación, análisis y traducción de unidades de naturaleza biosanitarias. Entreculturas, Revista 
de Traducción y Comunicación Intercultural 1: 385-408. http://www. entreculturas.uma.es/n1pdf/articulo20.pdf / [Acceso 25/03/2019].

Martínez-López, Ana Belén. 2009c.Terminología y fraseología en los documentos médico-legales (1): extracción, clasificación, análisis y traducción de unidades de naturaleza jurídica. Entreculturas, Revista de Traducción y Comunicación Intercultural 1: 409-424. http://www. entreculturas.uma.es/n1pdf/articulo21.pdf [Acceso 25/03/2019].

Mugüerza, Pablo, Barbetti Vros, Lida \& Gallego-Borghini, Lorenzo. 2011. Glosario crítico inglés-español de documentos de consentimiento informado.Panace@: Revista de Medicina, Lenguaje y Traducción 12(33): 19-34. http://www.tremedica.org/panacea/IndiceGeneral/n33Tradyterm-MuguerzaVrosBorghini.pdf [Acceso 04/04/2019].

Navarro, Fernando A. (2010). Estudios clínicos y ensayos clínicos. Laboratorio del lenguaje. https://medicablogs.diariomedico.com/laboratorio/ 2010/06/09/estudios-clinicos-y-ensayos-clonicos/[Acceso20/03/2018].

Navarro, Fernando A. 2017. Medizin. Gran diccionario médico alemán-español. Edición en línea (versión 1.02). Madrid: Cosnautas. https://www. cosnautas.com/es/catalogo/diccionario-aleman [Acceso 14/04/2019].

Navarro, Fernando A. 2019. Libro rojo. Diccionario de dudas y dificultades de traducción del inglés médico (3. ${ }^{\mathrm{a}}$ ed.). Edición en línea (versión 1.02). Madrid: Cosnautas. https://www.cosnautas.com/es/catalogo/librorojo [Acceso 14/04/2019].

Pajares Nievas, José Sergio. 2015. La traducción de textos médico-jurídicos: un breve análisis. FITISPos 5: 183-193. http://www3.uah.es/fitispos_ij/ OJS/ojs-2.4.5/index.php/fitispos/article/view/73/84 [Acceso 10/03/ 2019].

Pantoja Zarza, Lucía. 2004. El consentimiento informado: ¿sólo un requisito legal? Revista Española de Reumatología 31(8): 475-478. https://www. elsevier.es/es-revista-revista-espanola-reumatologia-29-pdf-13067152 [Acceso 10/03/2019].

Parcet, Mónica. 2018. Glosario (alemán-español) de términos relacionados con el sistema sanitario alemán.Panace@: Revista de Medicina, Lenguaje y Traducción 19(47) 6-21. http://www.tremedica.org/panacea/ IndiceGeneral/n47-terminologia-1.pdf [Acceso 10/04/2019].

Real Academia Española y Asociación de Academias de la Lengua Española.

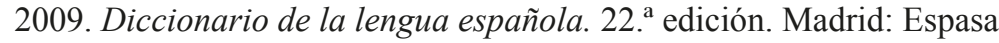
Calpe.

Real Academia Española y Consejo General del Poder Judicial. 2016. Diccionario del español jurídico. https://dej.rae.es/ [Acceso 12/04/2019]. 
Real Decreto 944/1978, de 3 de agosto, por el que se regulan los ensayos clínicos de productos farmacéuticos y medicinales. https://www.boe.es/eli/ es/o/1982/08/03/(1) [Acceso 08/04/2019].

Real Decreto 223/2004, de 6 de febrero, por el que se regulan los ensayos clínicos con medicamentos. https://www.boe.es/eli/es/rd/2004/02/06/223/ con [Acceso 08/04/2019].

Real Decreto 561/1993, de 16 de abril, por el que se establecen los requisitos para la realización de ensayos clínicos con medicamentos. https:/www. boe.es/eli/es/rd/1993/04/16/561 [Acceso 08/04/2019].

Rodes Lloret, Fernando. 2014. En Crimipedia, Centro Crímina para el Estudio y Prevención de la Delincuencia. http://crimina.es/crimipedia/ wp-content/uploads/2014/07/Medicina-legal-y-forense...pdf [Acceso 18/04/2019].

Saladrigas Iserin, María Verónica. 2019. DIC. Diccionario inglés-español de investigación clínica. Edición en línea (versión 1.04). Madrid: Cosnautas. https://www.cosnautas.com/es/catalogo/dic [Acceso 14/04/2019].

Silva Silva, Hernán. 2012. Medicina Legal. Chile: Editorial Jurídica de Chile.

Unión Europea. Reglamento (UE) 2016/679 del Parlamento Europeo y del Consejo, de 27 de abril de 2016, relativo a la protección de las personas físicas en lo que respecta al tratamiento de datos personales y a la libre circulación de estos datos y por el que se deroga la Directiva 95/46/ CE (Reglamento general de protección de datos). Diario Oficial de la Unión Europea L 119/1, 4 de mayo de 2016. https://eur-lex.europa.eu/ lega-content/ES/TXT/HTML/?uri=CELEX:32016R0679\&from=ES [Acceso 19/09/2019]. 